La

Révolution

française

\section{La Révolution française}

Cahiers de l'Institut d'histoire de la Révolution française

\section{$8 \mid 2015$}

Entre la Révolution et l'Empire : une nouvelle politique dans l'océan Indien

\title{
In memoriam - Stéphane Mandelbaum (1985 - 2014)
}

\section{Bernard Gainot}

\section{(2) OpenEdition}

Journals

Édition électronique

URL : http://journals.openedition.org/lrf/1311

DOI : $10.4000 /$ Irf. 1311

ISSN : 2105-2557

Éditeur

IHMC - Institut d'histoire moderne et contemporaine (UMR 8066)

Édition imprimée

Date de publication : 23 juin 2015

\section{Référence électronique}

Bernard Gainot, «In memoriam - Stéphane Mandelbaum (1985 - 2014) », La Révolution française [En ligne], 8 | 2015, mis en ligne le 24 juin 2015, consulté le 15 septembre 2020. URL : http:// journals.openedition.org/lrf/1311

Ce document a été généré automatiquement le 15 septembre 2020.

(c) La Révolution française 


\title{
In memoriam - Stéphane Mandelbaum (1985 - 2014)
}

\author{
Bernard Gainot
}

1 Stéphane est né le 9 octobre 1985. Il aurait eu 30 ans cette année.

Après son baccalauréat obtenu en 2003 au lycée Henri IV avec une mention "Très bien ", il est inscrit en hypokhâgne, puis en khâgne, dans le même lycée, de 2003 à 2006. Il intègre en 2006 l'Essec, où il va suivre un programme d'alternance de cours théoriques et de stages professionnalisants, au cours desquels il obtient des compétences variées, notamment en matière de modélisation financière, de gestion de bases de données, d'analyste-programmeur.

C'est cette même année 2006 que je le rencontre à l'Université Paris 1 PanthéonSorbonne. Passionné d'histoire, il ne se voyait pas entièrement immergé dans une carrière commerciale, aussi gratifiante et absorbante soit-elle, et il avait un besoin vital de «s'oxygéner », me disait-il, en préparant un master de recherche.

2 Les détours par lesquels il prit contact avec moi sur le terrain de l'histoire impériale de la France moderne, me sont restés inconnus. Toutefois, son ouverture remarquable sur des pays aussi divers que la Chine, le Mexique, l'Indonésie, le Japon, qu'il avait pu explorer au cours de ses multiples voyages, le prédisposaient à parcourir des espaces éloignés dans le temps et dans l'espace, avec un esprit neuf et un questionnement permanent.

3 Il a choisi pour son mémoire de M1 une expédition largement inconnue, mais éminemment fondatrice, celle que le gouverneur de la Guyane Victor Hugues, (bien connu par ailleurs pour avoir apporté le décret d'abolition de l'esclavage, et pour avoir ensuite mené une carrière révolutionnaire, en Guadeloupe, de 1794 à 1798) a mise sur pied conjointement avec le gouverneur du Sénégal Blanchot pour reprendre aux Anglais l'île de Gorée en nivôse an XII (décembre 1804). Ce travail s'inscrivait dans une problématique, que nous avions définie d'un commun accord: l'émergence d'une conception impériale (c'est-à-dire intégrée) à travers la conduite d'opérations militaires combinées transversales par rapport à l'ancien modèle mercantiliste centré sur la métropole. En l'espèce, il s'agit de la relation transatlantique Amérique 
tropicale/Afrique occidentale, mais d'autres circuits étaient mentionnés dans le travail de Stéphane : la relation Antilles/Canada par voie maritime, ou mieux encore par la voie du Mississipi à travers la Nouvelle-Orléans; ou le riche tissu relationnel qui s'élabore après la perte de Pondichéry, autour de Port-Louis dans l'île-de-France (actuelle île Maurice), tout autour de l'Océan Indien, tissu où le commercial se distingue mal du militaire, singulièrement activé dans les périodes de conflit (1778-1783, puis 1795-1810) où la rupture du lien avec la métropole oblige à reconfigurer les réseaux.

4 Pour son M2, je demandai donc à Stéphane de repartir de son étude de cas et de ses hypothèses pour opérer une remontée dans le temps et une dilatation des espaces. Il fit ainsi remonter l'émergence de la nouvelle conception « impériale » aux lendemains de la Guerre de Sept Ans. Il a dépouillé les fonds de la correspondance, tant au départ qu'à l'arrivée, tant au CARAN à Paris, qu'aux archives de l'outre-mer à Aix-en-Provence, pour les colonies suivantes: Sénégal, Martinique, Louisiane, Canada, Guyane. Il était absolument passionné pour les Souvenirs maritimes de Scipion de Castries, ouvrage qui avait suscité chez lui autant d'interrogations que d'enthousiasme, ce qui transpire au fil des pages à travers les multiples notes de références. Nous avions même envisagé la possibilité d'en proposer une édition critique. Nous nous étions mis d'accord pour que Stéphane mette au point ses outils pour l'analyse de l'espace atlantique, tandis que je commencerai à explorer parallèlement les sources pour l'Océan Indien, espace que j'avais jusqu'alors très peu abordé, à partir de la volumineuse correspondance du gouverneur des comptoirs des Indes orientales Charles Decaen (1803-1810).

5 La caractéristique majeure de la mise en réseau des interrelations dans l'Empire est le passage d'une organisation «classique» type colbertiste stellaire, centrée sur la métropole, à une organisation plus complexe, de type réticulaire, avec des interconnexions multiples à partir des bases navales stratégiques et des entrepôts coloniaux comme Le Cap à Saint-Domingue, La Nouvelle-Orléans, Fort-de-France, mais aussi avec des relais dans les territoires alliés ou neutres comme La Havane, Boston, Le Cap en Afrique du Sud. Ce système élaboré à partir de la cartographie des liaisons maritimes, s'appuie sur une historiographie regroupant des synthèses historiques comme celles de Christian Buchet sur les conflits dans l'espace caraïbe à la période moderne, de Patrick Villiers sur l'imbrication de la guerre et du commerce à l'époque de la Guerre d'Indépendance américaine; ou bien des travaux de modélisation, et notamment le très riche essai d'anthropologie stratégique de Philippe Forget et Gilles Polycarpe, le réseau et l'infini, qui a beaucoup servi pour proposer une réponse aux hypothèses avancées dans le mémoire de l'année précédente.

6 Le mémoire de M2, actuellement disponible sur place à la bibliothèque de l'Institut d'histoire de la Révolution française de l'Université Paris 1 (cote : Z 1042), qui était notre laboratoire commun de recherche, se divise en deux parties. Une première partie présente l'espace-temps de l'information; c'est la guerre qui désorganise le système stellaire traditionnel. De nouvelles routes s'ébauchent, les temps pour parcourir les mêmes distances diminuent considérablement (de deux mois à trois semaines en moyenne). La déstructuration doit beaucoup à l'ordonnance royale du 31 juillet 1763 , ordonnance portant réglement au sujet des paquebots destinés à la correspondance des colonies. L'Etat se désengage, pour des raisons financières, couvertes par des justifications de vitesse, du service de la Poste vers les colonies. Il y a une externalisation de la gestion des lettres et des paquets pour les navires de commerce. Parallèlement, de nouveaux acteurs apparaissent, comme les réseaux diplomatiques, ou 
comme les réseaux de renseignement. En temps de guerre, les opérations sont articulées autour de l'effet de surprise, qui a une dimension aussi bien tactique que stratégique. Les convois et les escadres en sont déstabilisés mais, pour les populations, le sentiment d'appartenance se renforce, jusqu'à réaliser une intégration véritable aux identités culturelles multiples toutefois : «Les colonies émergent comme des acteurs propres, se reconnaissent comme françaises en dépit de la rupture des communications » (p. 87). Cette émergence de la créolisation autour d'un sentiment d'appartenance est un phénomène culturel majeur de la deuxième partie du XVIII ${ }^{\mathrm{e}}$ siècle dans les empires américains.

7 La deuxième partie analyse les mutations du commandement à travers les correspondances administratives. Compte tenu des distances et de l'évolution des relations, la délégation de pouvoir est indispensable; mais d'un autre côté, en cas de conflit, l'unité de commandement s'avère indispensable. Il faut donc recourir de plus en plus souvent aux opérations combinées entre forces de terre et forces de mer comme méthode de résolution de la contradiction. «Les choses se lient tellement dans les expéditions d'outre-mer que les deux armes ne peuvent point être tout à fait étrangères l'une à l'autre " (p. 89). Ces opérations "amphibies" ou combinées projettent outre-mer des troupes de diverses origines, qui doivent s'adapter au terrain, mais aussi s'articuler les unes aux autres.

Outre les régiments de l'armée de terre, et les troupes de marine, il faut avoir recours aux supplétifs autochtones, aux milices de tous types. Pour ces dernières, l'intégration est plus problématique, au moins pour les troupes amérindiennes de l'Amérique septentrionnale, puisque, comme le dit le gouverneur de la Louisiane Kerlerec « la force de la parole française ne consiste que dans les marchandises qui l'accompagnent. »

Il y a un type de troupes intégrées, en revanche, qui répondent parfaitement aux visées opérationnelles des expéditions outre-mer, ce sont les troupes de marine, héritières des compagnies franches de la Marine détachées dans le Nouveau-Monde par Richelieu. Ce sont les soldats du nouveau réseau en formation. Or, paradoxe français, ces troupes sont supprimées en 1761, alors que les Britanniques vont y avoir de plus en plus recours. Ces soldats mobiles peuvent intervenir dans tous les espaces, à la mesure du système qui se met en place pour l'Empire britannique, selon lequel la mer est une opportunité, et non un obstacle comme pour les Français. La démonstration recourt une fois encore à Forget et Polycarpe (Le réseau et l'infini) p. 107 : « L'Empire britannique ne subit pas un espace donné, il élabore la mer, s'en fait la cause efficiente. Il ne s'agit pas de caboter de côte en côte, mais de s'approprier la surface des eaux en traçant extensivement sur elle des chemins virtuels et réitérables de connexion.»

Outre les difficultés structurelles d'articulation, le commandement français souffre d'une division entre deux autorités qui relèvent de cultures différentes, de la persistance d'un esprit de corps attaché aux particularismes des organismes d'origine. Je pourrai avancer comme hypothèse que cela renvoie aux représentations et à l'organisation des domaines d'intervention de l'Etat à l'âge classique, comme fragments autonomes mais interagissants d'une grande machine organique. Stéphane préfère développer des exemples concrets pour illustrer ces oppositions récurrentes : le viceamiral Charles d'Estaing face au marquis de Bouillé en 1779-1780; le marquis de Montcalm face au marquis de Vaudreuil au cours de la Guerre de Sept Ans au Canada; le gouverneur de Louisiane Kerlerec face à l'intendant Rochemore lors de la Guerre d'Indépendance américaine. Cette dyarchie mal harmonisée est confrontée au 
système britannique de responsabilisation du commandement, mise en place avec les Articles of War de 1749. La nouveauté et la radicalité du système né de l'application de ce dispositif réglementaire sont longuement et pertinemment soulignées (p. 146 et alii). Mais la démonstration ne se termine absolument pas sur ce constat de la supériorité navale britannique, finalement assez convenu, qui sature les histoires maritimes comparées de la France et de la Grande-Bretagne. Stéphane souligne au contraire toutes les avancées vers le nouveau système réticulaire qui se font jour dans ce qui va devenir de plus en plus l'Empire français, de 1763 à 1810 ; l'affirmations de véritables carrières coloniales, avec la mobilité des hommes (les Vaudreuil, le marquis de Dumas, La Galissonière, Victor Hugues, etc...) ; la voie spécifique dans le cas français avec l'intégration poussée du commerce et du militaire (les nombreuses campagnes de guerre au commerce qui ont souvent pris en défaut les forces britanniques), qui est parallèle à l'intégration politique par l'intégration militaire (l'esquisse d'une véritable République impériale par l'égalisation des conditions de ses populations).

11 Les fortes conclusions de ce travail soulignent sa dimension historiographique par une prise de distance très nette envers les courants dominants essentiellement descriptifs de l'atlantic history:

12 Les évolutions techniques et commerciales, l'intensification des échanges dans le monde rapprochent très certainement les espaces et les hommes à la fin du XVIII siècle. Or, ce rapprochement n'est pourtant pas le signe annonciateur de l'avènement d'une nouvelle civilisation multiculturelle, comme le pensent les premiers historiens de l'atlantic history. Bien au contraire, cette intégration économique de plus en plus poussée entraîne une militarisation de ces espaces et l'avènement de nouveaux conflits. Loin d'être de simples accidents de l'Histoire, les guerres coloniales du XVIII siècle sont donc étroitement liées au développement du commerce. On ne saurait, dès lors, envisager l'histoire des espaces coloniaux sans penser ce conflit entre les nations pour leur appropriation." (p. 165)

Un regard aussi neuf, une perspective aussi clairement formulée, nécessitent le temps de la critique et de la réflexion. Ils remettent en cause les routines de l'académisme, encore bien installé comme j'ai pu m'en apercevoir à la lecture du rapport sur le travail de Stéphane, que j'avais présenté pour le prix d'histoire militaire, et qui fut repoussé sous des prétextes qui masquaient mal le désarroi d'une pensée conservatrice déroutée par l'ampleur de la réflexion.

14 Au cours de ces deux années de participation au séminaire d'histoire militaire de la période moderne, que je dirigeais avec Hervé Drévillon et Benjamin Deruelle, Stéphane a su se faire apprécier de ses nombreux camarades par sa gentillesse, son humour, et sa très grande simplicité ; qualités remarquées notamment lors du voyage d'études dans le Val d'Aoste au printemps 2008, et qui sont durablement inscrites dans les souvenirs de tous les participants. Sa très vaste culture, dans des domaines aussi variés que la littérature, le cinéma, ou la musique, facilitaient grandement les échanges. Il était totalement fasciné par les romantiques allemands, qui viennent parfois habiter son travail comme ils habitaient ses illusions. Ainsi Jean-Paul apparait-il incidemment, mais non gratuitement, au détour d'un paragraphe (p. 24) : «Il suffit d'une guerre dans un pays pour avoir aussitôt de meilleures informations sur sa géographie. »

15 Après 2008, il y eut une période d'éloignement, Stéphane ayant occupé des postes lointains, notamment à Hong-Kong et à La Nouvelle-Orléans. Mais nous avions renoué lorsqu'il était venu me faire part de son désir de commencer une thèse en Histoire. 
Comme je ne pouvais plus le diriger d'un point de vue administratif, je lui avais proposé de s'intégrer progressivement aux activités de l'Université Paris 1, soit dans le cadre de l'Institut d'histoire de la Révolution française, soit dans le cadre du nouvel Institut sur la Guerre et sur la paix, dont il connaissait par ailleurs quelques-un(e)s des participants. En attendant, nous avions préparé ensemble une communication au colloque sur la Guerre de Sept Ans, organisé par Marion Godfroy, Hervé Drévillon et Pierre Serna. Cette communication était envisagée comme le point de départ d'une publication écrite plus vaste sur l'émergence de l'Empire entre 1750 et 1810 . Nous nous étions revus plusieurs fois pour échanger à ce propos, et Stéphane se projetait constamment dans l'avenir.

Et puis le destin est venu foudroyer ces perspectives, comme si un trop-plein d'attente était venu se fracasser sur un réel trop prosaïque.

Que non seulement sa mémoire, mais ses écrits, perdurent. 